\title{
A Simple Model for Reflection-Driven Spectral Evolution of Turbulence in the Corona and Inner Heliosphere
}

\author{
T. Laitinen \\ Väisälä Institute in Space Physics and Astronomy, University of Turku, Finland
}

Received: 7 March 2005 - Revised: 7 September 2005 - Accepted: 2 October 2005 - Published: 20 December 2005

\begin{abstract}
Dissipation of turbulence is generally considered as a prime candidate for the heating and acceleration of solar wind from the corona throughout the heliosphere, due to radially evolving dissipation processes and/or cascading of energy to dissipation scales. In order to model the latter consistently, we must consider the evolution of cross-helicity when modeling the strength of the cascade, as the non-linear interactions causing it require counterstreaming waves. In this paper, we present a simple model of non-WKB wave reflection from large-scale gradients, and apply the resulting cross-helicity to evaluate the evolution of the turbulence spectrum up to $0.3 \mathrm{AU}$ by using a phenomenological cascade model. The study is restricted to a cascade in perpendicular direction. We study the ability of this spectral flux to heat the solar wind, and its dependence on the frequency of the excited waves. We find that the cascade advances fast in the low corona, and has a strong frequency dependence. The heating rate is high close to the coronal base, but decreases very quickly. In the corona, the heating rate varies by an order of magnitude in the frequency range of $10^{-6}$ to $10^{-3} \mathrm{~Hz}$, suggesting that the shape and range of the frequency spectrum has important implications on the solar wind modeling.
\end{abstract}

\section{Introduction}

Solar wind heating remains an unsolved problem, nearly five decades after the need for an extremely hot corona to obtain the high solar wind speeds was first postulated by Parker (1958). The acceleration of the solar wind requires that the heat deposition must continue above the sonic point, at heights above a few solar radii (e.g. Leer et al., 1982). At these heights, however, the low Coulomb collision rates render collisional heating methods weak, and the collisionless dissipation processes of plasma waves, capable of transporting energy from the Sun to the corona, have gained much

Correspondence to: T. Laitinen (timo.laitinen@ srl.utu.fi) support in the theoretical research (for review, see, e.g. Cranmer, 2002).

For the origin of such waves, Axford and McKenzie (1992) suggested that small-scale reconnection events in the supergranular network could excite high-frequency waves that could escape to the corona, to be damped there by ion cyclotron resonance. This mechanism was modeled by $\mathrm{Tu}$ and Marsch (1997), who were capable of producing reasonable solar wind profiles. Lately, some criticism has been directed to the validity of the model, due to the erosion of the spectrum at lower frequencies by minor ions (e.g. Cranmer, 2000). Also, the required high-frequency waves remain still beyond observational resolution. The observations of the temperature anisotropies in the corona (Kohl et al., 1998) and the particle velocity distributions in the interplanetary space (Marsch and Tu, 2001), however, support the idea that the ion cyclotron resonance at least partly participates in the solar wind dynamics.

As an alternative approach, it has been suggested that the energy is cascaded non-linearly from larger scales to the dissipative scales (see, e.g. Tu and Marsch, 1995, and references therein). Hollweg (1986) presented a model where the solar wind is heated by a cascade at the Kolmogorov energy transfer rate. Hu et al. (1999) combined the evolution of the spectral shape of the turbulence studied by Tu et al. (1984) and Tu (1988) with the ion cyclotron mechanism presented by Tu and Marsch (1997). These studies, however, do not take into account the anisotropic nature of the turbulent cascade of Alfvén waves in a strong magnetic field, which has been recently studied theoretically (e.g. Goldreich and Sridhar, 1995) and through simulations (e.g. Shebalin et al., 1983; Cho and Vishniac, 2000). To this end, Matthaeus et al. (1999) have presented a reduced MHD model, which incorporates a purely perpendicular cascade of low-frequency waves to an unspecified dissipation process at high perpendicular wave numbers. Such a models are, however, limited to small heliospheric distances due to computational demand. Verdini et al. (2004) and Cranmer and van Ballegooijen (2005) model the heating by wave transport equations and 
heating by cascade into small scales. They do not, however, consider the resulting evolution of the spectral shape. Cranmer and van Ballegooijen (2003), on the other hand, have presented a phenomenological model of an anisotropic cascade, following the spectral evolution models by $\mathrm{Tu}$ et al. (1984), Tu (1988) and Zhou and Matthaeus (1990a).

The phenomenological models, while convenient and relatively fast, typically suffer from one considerable shortcoming with respect to consistency. The existence of wave-wave interactions requires waves traveling with a non-zero velocity relative to each other, which, for dispersionless Alfvén waves, amounts to waves traveling in opposite directions along the magnetic field. While this cross-helicity determines the strength of the energy cascade, the phenomenological models tend to use simple approximations instead of a consistent approach. In this paper we will implement a simple model for the evolution of the cross-helicity due to non-WKB reflections, as presented by Heinemann and Olbert (1980), and applied by Verdini et al. (2004) and Cranmer and van Ballegooijen (2005) in their studies. Crosshelicity is used to obtain the phenomenological evolution of the turbulence spectrum due to cascade in perpendicular direction. The model is used to estimate the heat flux produced by dissipation of the turbulence, without specifying the dissipation mechanisms. The heating profiles are compared with the empirical heating profiles given by Lionello et al. (2001) and Lie-Svendsen et al. (2003), and the modeled profile of Hu et al. (1999).

The paper is organized as follows: In Sect. 2, we present the models used in the study. We use a simple solar wind model, presented in Sect. 2.1. The non-WKB reflections are solved from Elsasser equations and converted to power spectral units in Sect. 2.2. The perpendicular spectral flux is presented and a solution method is derived in Section 2.3, and the heating rate is given in Sect. 2.4. The results are given and discussed in Sect. 3 and the conclusions are drawn in Sect. 4.

\section{The Model}

\subsection{Solar wind model}

While it would be possible to obtain the solar wind solution by integrating numerically the hydrodynamic conservation equations, we find it to be beyond the scope of the current study. Instead, in this work, we obtain the solar wind profiles by using an empirical estimate for the electron density profile,

$\frac{n_{e}}{n_{e 0}}=\frac{1}{\hat{r}^{2}}+\frac{25}{\hat{r}^{4}}+\frac{300}{\hat{r}^{8}}+\frac{1500}{\hat{r}^{16}}+\frac{5796}{\hat{r}^{33.9}}$,

with $\hat{r}=r / r_{\odot}$, where $r_{\odot}$ is the solar radius and $n_{e 0}=1.35 \cdot 10^{5} \mathrm{~cm}^{-3}$, as given by Cranmer and van Ballegooijen (2005), and the magnetic field configuration

$B(r)=B_{0}\left(\frac{r_{0}}{r}\right)^{2}\left[1+1.9 b_{f}\left(\frac{r_{0}}{r}\right)^{6}\right]$,

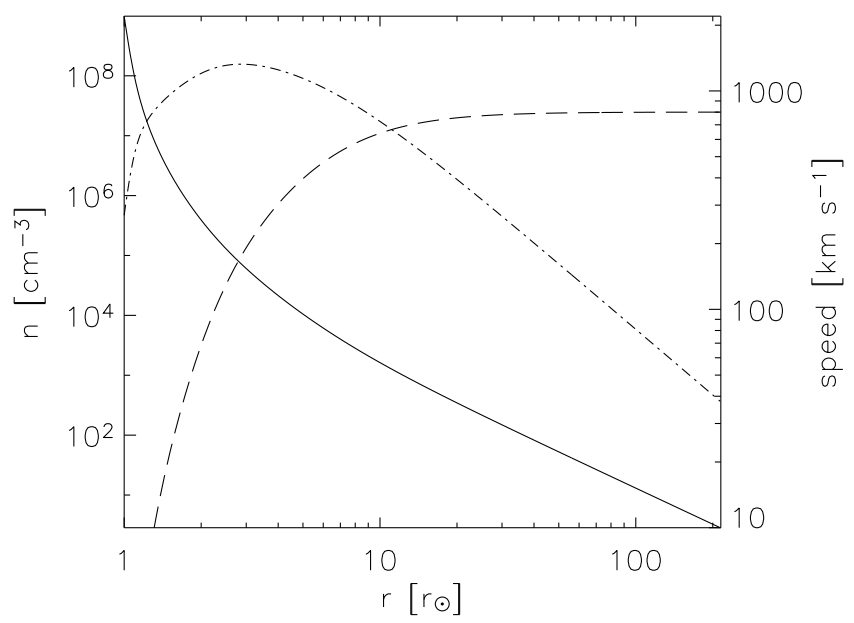

Fig. 1. Solar wind number density and velocity profiles used in the study. The density profile is shown by the solid curve, while the dashed and dash-dotted curves represent the solar wind and the Alfvén speed, respectively. The heliocentric distance is presented in units of solar radius, $r \odot$.

allowing for superradial expansion close to the coronal base defined by the parameter $b_{f}$, for which we use in this study $b_{f}=1$. Using these profiles, and the mass conservation equation, $n_{e} U / B=$ const, we can obtain the profiles for the flow velocity, $U$, and the Alfvén velocity, $V_{A}=B / \sqrt{4 \pi m_{p} n_{e}}$, given a reference value for the velocity and the magnetic field strength at a known point. In this work, we consider $U_{0} \equiv U(1 \mathrm{AU})=800 \mathrm{~km} / \mathrm{s}$ and $B_{0} \equiv B$ $(1 \mathrm{AU})=2.9 \cdot 10^{-5} \mathrm{G}$. With these choices, the coronal base magnetic field strength is $3.9 \mathrm{G}$, and the Alfvén velocity reaches its maximum, $1326 \mathrm{~km} / \mathrm{s}$, at heliocentric altitude of $2.9 r_{\odot}$. The solar wind profiles are shown in Fig. 1.

\subsection{Turbulence model}

\subsubsection{Elsasser variables}

When studying small-scale disturbances in a slowly varying background plasma, it is customary to linearize the corresponding equations for the flow velocity and magnetic field strength as $\mathbf{U}_{\text {tot }}=\mathbf{U}+\mathbf{u}$ and $\mathbf{B}_{\text {tot }}=\mathbf{B}+\mathbf{b}$. As is well known, the MHD equations can be reformatted for incompressive fluctuations into a pair of Elsasser equations (Elsasser, 1950; Marsch and Mangeney, 1987), describing the motion of disturbances traveling parallel and anti-parallel to the magnetic field. In this work, we define Elsasser variables in magnetic field units, $\mathbf{z}_{ \pm}=\mathbf{u} \sqrt{4 \pi \rho} \pm \mathbf{b}$, where $\rho$ is the plasma density, instead of the more conventional velocity units. This selection is motivated by an other field of research where the current research may be used, namely studies of energetic particles, where the magnetic fluctuations determine the energetic particle transport and acceleration. While the derivation of the Elsasser equations is straightforward, it is somewhat lengthy, and we will not repeat it here. For treatment with the velocity units, see, e.g. Marsch and Tu (1989); 
Zhou and Matthaeus (1990b), or in magnetic field units in radial geometry, Barkhudarov (1991). Using the magnetic field units, toroidal waves propagate in cylindrically symmetric geometry as defined by equation

$$
\frac{\partial z_{\phi}^{ \pm}}{\partial t}+V_{\mp} \frac{\partial z_{\phi}^{ \pm}}{\partial r}-V_{\mp} z_{\phi}^{ \pm} \frac{\partial}{\partial r}\left(\ln z_{W}^{ \pm}\right)+V_{ \pm} R_{A} z_{\phi}^{\mp}=N_{\phi}
$$

where $z_{\phi}^{ \pm}$are the toroidal components of the Elsasser variables, $V_{\mp}=U \mp V_{A}$ with $V_{A}=B / \sqrt{4 \pi \rho}$ the Alfvén velocity. The wave propagation and evolution is determined by the convection term, and the WKB and reflection terms, where

$z_{W}^{ \pm}=\frac{V_{\mp}\left(r_{0}\right)}{V_{\mp}} \sqrt{\frac{A\left(r_{0}\right)}{A} \frac{V_{A}}{V_{A}\left(r_{0}\right)}} \quad$ and

$R_{A}=-\frac{1}{2} \frac{\partial}{\partial r} \ln V_{A}$,

and $A \propto 1 / B$ is the flux tube cross-section. The nonlinear interactions are presented by the variable $N_{\phi}$, and will be considered separately in a phenomenological manner.

In the absence of reflections and nonlinear interactions, the solution of the equation is given as

$z_{\phi}^{ \pm}(r, t)=z_{W}^{ \pm}(r) z_{\phi}^{ \pm}\left(r_{0}, t-r / V_{\mp}\right)$.

In the general case, however, the equation cannot be solved analytically. In Appendix A we present a semi-analytic method for the solution of the equation in the absence of nonlinear interactions, but with the reflections included. As a method of verification, we also apply the method introduced by Barkhudarov (1991). As the latter method proved both faster and more stable around the Alfvénic point, we apply the Barkhudarov's method in further analysis.

Using the simplified form of Eq. (2), given in Eq. (A1), we can study the general behaviors of the solution. The two equations can be decoupled by derivation to give secondorder differential equations

$\frac{\partial^{2} z_{0}^{ \pm}}{\partial s^{2}}=z_{0}^{ \pm} \pm i \omega \frac{\partial \tau_{\phi}}{\partial s} \frac{\partial z_{0}^{ \pm}}{\partial s}=z_{0}^{ \pm} \pm i \frac{\omega}{\omega_{c}} \frac{\partial z_{0}^{ \pm}}{\partial s}$,

where $d s=R_{A} d r, z_{0}^{ \pm}$and $\tau_{\phi}$ are the normalized forms of the Elsasser variables and the transit time difference between these variables, respectively, as given in Appendix A. The characteristic frequency for the reflection, $\omega_{c}$, is thus, following the definition of $\tau_{\phi}$ in Appendix A,

$$
\left|\omega_{c}\right|=\left|\frac{\partial \tau_{\phi}}{\partial s}\right|^{-1}=\left|\frac{1}{R_{A}} \frac{2 V_{A}}{U^{2}-V_{A}^{2}}\right|^{-1} .
$$

It can be readily seen that the behavior of the equation is determined by the frequency of the wave, relative to $\omega_{c}$. On high-frequency limit, the equation reduces into $\partial z_{0}^{ \pm} / \partial s=0$, which produces simply the WKB solution, and reflection vanishes. On the low-frequency limit, the first-order term vanishes and, using $z_{\phi}^{ \pm}=z_{C}^{ \pm} z_{W}^{ \pm}$, the resulting equation can be returned into form

$\frac{\partial z_{C}^{ \pm}}{\partial r}+R_{A} z_{C}^{\mp}=0$ which describes strong, frequency-independent reflection. Thus, it is clear that if the strength of the nonlinear interactions depends on the ratio of waves traveling parallel and anti-parallel to the background magnetic field, the frequency range and spectrum of the excited waves is an important parameter for the turbulence studies.

\subsubsection{Spectral domain}

The standard method of obtaining the wave evolution in the spectral domain is to multiply Eq. (2) with $z_{\phi}^{ \pm}\left(r+r^{\prime}\right)=z_{\phi}^{\prime \pm}$ and $z_{\phi}^{ \pm}$at locations $r$ and $r+r^{\prime}$, respectively, and form a sum of the equations (Marsch and Tu, 1989; Zhou and Matthaeus, 1990b). Then, integrating over the domain to obtain the correlation functions, and Fourier-transforming the result yields

$$
\begin{aligned}
\frac{\partial P_{ \pm}}{\partial t} & +V_{\mp} \frac{\partial P_{ \pm}}{\partial r}-k \frac{\partial V_{\mp}}{\partial r} \frac{\partial P_{ \pm}}{\partial k} \\
& -V_{\mp} P_{ \pm} \frac{\partial}{\partial r} \ln \left\{P_{W \pm}\right\}+2 V_{ \pm} R_{A} F_{\mp}=0,
\end{aligned}
$$

where $P_{ \pm}$are the Fourier transforms of the correlations $\left\langle z_{\phi}^{ \pm} z_{\phi}^{\prime \pm}\right\rangle, P_{W \pm}=\left(z_{W}^{ \pm}\right)^{2}$, and the mixing term, $2 F_{\mp}$, is the Fourier transform of $\left\langle z_{\phi}^{+} z_{\phi}^{\prime-}+z_{\phi}^{\prime+} z_{\phi}^{-}\right\rangle$.

This form of the equation is, however, unnecessarily complicated. The convective term in wave number space turns the equation into a 2D-equation. Another complication results from the fact that the reflection process conserves the wave frequency in the frame fixed to the Sun. Therefore, when attempting to formulate a simple approximation for the mixing term, one should take into account the fact that the mixing occurs between waves of different scales.

We take an alternative route to overcome these problems, and perform the correlations and Fourier transforms in the temporal domain. In this case, the third term of the previous equation vanishes, and the equation is reduced to a 1D equation.

For the mixing term, we consider a simple closure model. If we consider the Fourier presentation $z_{\phi}^{ \pm}(r, t)=z_{\phi 0}^{ \pm}(r, \omega) e^{-i \omega t}$, we find that the correlations in the mixing term are of the form $z_{\phi 0}^{+} z_{\phi 0}^{-} S_{ \pm}(r, \omega)$, where $S_{ \pm}$is a complex variable. While the mixing is clearly connected to the $S_{ \pm}$variables, we seek a simpler description, using the frequency dependence of the reflection process, described above, and formulate the mixing term as $F_{\mp}=-R_{\omega} \sqrt{P_{ \pm} P_{\mp}}$, where $R_{\omega}(r, \omega)$ describes the frequency dependence of the reflection process. Then, using $P=P_{C} P_{W}$ we have

$\frac{\partial P_{C \pm}}{\partial t}+V_{\mp} \frac{\partial P_{C \pm}}{\partial r}-2 V_{ \pm} R_{A} R_{\omega}\left|\frac{V_{\mp}}{V_{ \pm}}\right| \sqrt{P_{C \pm} P_{C \mp}}=0$,

or, in a steady state form,

$\frac{\partial P_{C \pm}}{\partial r}-2 R_{A} R_{\omega} \operatorname{sign}\left\{V_{-}\right\} \sqrt{P_{C \pm} P_{C \mp}}=0$.

This can be solved by using $Q^{2}=P$ to get

$2 Q_{C \pm} \frac{\partial Q_{C \pm}}{\partial r}-2 R_{A} R_{\omega} \operatorname{sign}\left\{V_{-}\right\} Q_{C \pm} Q_{C \mp}=0$, 
which is similar to Eq. (5), and can be formally solved as

$Q_{C \pm}=Q_{0 \pm}+\int_{r_{0}}^{r} \operatorname{sign}\left\{V_{-}\right\} R_{\omega} R_{A} Q_{C \mp} \mathrm{d} r$

except for the singular point $Q_{C+}\left(r_{A}\right)=0$.

Similarly to the frequency dependence of the reflection efficiency shown by Eq. 3, also the reflection parameter $R_{\omega}(r, \omega)$ should exhibit constant value at low frequencies, compared to $\omega_{c}$, and decay to small values at higher frequencies, diminishing the effect of the reflection to the wave fields. While it would be possible to devise an approximate form for $R_{\omega}$ with such behavior, in this work it is not needed. Instead, we can make use of the solution of the Elsasser equations to obtain the fields $z_{\phi}^{ \pm}$for a given frequency, and subsequently, from the Fourier representation of the fields, the spectral variable $P$. This could be then used to solve the frequency dependence $R_{\omega}$ from Eq. (7), if needed. However, even this is not necessary, as in our further treatment the solutions $P_{ \pm}$are sufficient to provide all necessary information for the current study.

\subsection{Perpendicular cascade}

There are a number of ways to model the cascading in the heliosphere (see, e.g. Tu and Marsch, 1995). We follow the formulation by Zhou and Matthaeus (1990a), where the spectral evolution of the turbulence, due to nonlinear interactions, can be presented phenomenologically in terms of the spectral flux $F$ as

$$
\left(\frac{\partial P_{ \pm}}{\partial t}\right)_{\mathrm{nl}}=-\frac{\partial F}{\partial k}=-\frac{\partial}{\partial k}\left(\frac{V_{A}}{B} k^{5 / 2} P_{\mp}^{1 / 2} P_{ \pm}\right) .
$$

The effect of the counterstreaming waves is often presented with the notation $\alpha_{ \pm}\left(r, \omega, k_{\perp}\right)=\sqrt{P_{\mp} / P_{ \pm}}$. In this study, we make the simplifying approximation that changes in $\alpha_{ \pm}$ are only due to reflection, and the parameters depend only on the wave frequency, i.e., the parallel wave number. As we further assume that the spectral flux advances only to smaller perpendicular scales, the parameter $\alpha_{ \pm}$can be extracted from the $k$-derivative. In the rest of the paper, the parallel extent of the wave number is referred by the associated frequency only.

In this formulation, the wave-wave interactions are considered to take place between waves of the same scale, i.e. the same wave number. However, as the wave phase speeds of the components differ, and change throughout the heliosphere, the frequencies of the waves are not equal for a fixed wave number, but obey

$\omega_{\mp}=\omega_{ \pm} V_{ \pm} / V_{\mp}$

While this effect is negligible close to the Sun and far in the interplanetary space, near the Alfvénic point the effect can be considerable, as the frequency of an inward wave for any given wave number approaches zero. As these very low-frequency inward waves originate (by reflection) from very large scale waves generated near the sun, of which the observations are more difficult to obtain, we cannot adequately model this effect, and hence will neglect the shift in this study.

Using the spectral evolution to complete the full wave transport equation, we obtain, in WKB units,

$$
\begin{aligned}
\frac{\partial P_{C \pm}}{\partial r} & -2 R_{A} R_{\omega} \operatorname{sign}\left\{V_{-}\right\} \alpha_{ \pm} P_{C \pm} \\
& =-\frac{\sqrt{P_{W \pm}}}{V_{\mp}} \frac{\partial}{\partial k_{\perp}}\left(\frac{V_{A}}{B} k_{\perp}^{5 / 2} \alpha_{ \pm} P_{C \pm}^{3 / 2}\right) .
\end{aligned}
$$

By estimating the parameter $\alpha_{ \pm}$as a parametric solution of the Eq. (2), the solution for vanishing spectral flux, $P_{C 0 \pm}$, can be written as

$$
P_{C 0 \pm}\left(r, \omega, k_{\perp}\right)=P_{0} \exp \left\{\int d r 2 R_{A} R_{\omega} \operatorname{sign}\left\{V_{-}\right\} \alpha_{ \pm}\right\},
$$

where $P_{0} \equiv P_{0}\left(r_{\odot}, \omega_{0}, k_{0 \perp}\right)$. The numerical values for these solutions will be obtained from the solution of the Elsasser equations, discussed above.

Using the above assumptions, Eq. (9) can be solved analytically using a similar derivation to the ones presented by Tu et al. (1984) and Vainio et al. (2003). In the derivation and further treatment, we consider the reflection, cascade and heating processes at one given frequency, and thus the spectrum is considered to depend only on distance and wave number.

The wave transport equation, Eq. (9), can be written in dimensionless form as

$\frac{\partial I}{\partial \tau}-I^{1 / 2} \frac{\partial I}{\partial x}=0$

where the superscripts denoting the wave mode have been dropped for clarity, as no cross-terms exist. The independent variables are

$x=\left(\frac{k_{n \perp}}{k_{\perp}}\right)^{2 / 3}$
$\tau=k_{n \perp}^{3 / 2} \int d r \alpha \frac{V_{A}}{V} \frac{P_{C 0}^{1 / 2} P_{W}^{1 / 2}}{B}$,

where $k_{n}$ is an arbitrary normalization wave number, and the dependent variable

$I=\frac{k_{\perp}^{5 / 3} P_{C}\left(k_{\perp}, r\right)}{k_{n \perp}^{5 / 3} P_{C 0}}=x^{-5 / 2} \frac{P_{C}\left(k_{n \perp} x^{-3 / 2}, r\right)}{P_{C 0}}$.

The equation can be formally solved as

$I(x, \tau)=I\left(x+I(x, \tau)^{1 / 2} \tau, 0\right)$.

From the form of the solution it can be clearly seen, that for low wave numbers, with $x \gg I(x, \tau)^{1 / 2} \tau \approx I(x, 0)^{1 / 2} \tau$, the initial solution is conserved. For a power law initial spectrum, $I(x, 0)=x^{-s}$, we find for this limit

$x \gg x_{c} \equiv \tau^{1 /(1+s / 2)}, \quad$ or

$k_{\perp} \ll k_{c \perp} \equiv k_{n \perp} \tau^{-3 /(2+s)}$. 
For high wave numbers, it is easy to see that the asymptotic solution is independent of variable $x$. Then, for a power law initial spectrum $I(x, 0)=x^{-s}$, we find the asymptotic solution

$I(x, \tau)=\tau^{-s /(1+s / 2)}=x_{c}^{-s}$,

or

$P_{C 0} x_{c}^{-s} x^{5 / 2}=P_{W} P_{C 0}\left(\frac{k_{n \perp}}{k_{c \perp}}\right)^{(5-2 s) / 3}\left(\frac{k_{c \perp}}{k_{\perp}}\right)^{5 / 3}$,

at wave numbers $x \ll I(x, \tau)^{1 / 2} \tau=\tau^{1 /(1+s / 2)}=x_{c}$.

Thus, simplifying with $q=(5-2 s) / 3$, we have the asymptotic solutions

$P(r, k \perp)= \begin{cases}P_{W} P_{C 0}\left(\frac{k_{n \perp}}{k_{\perp}}\right)^{q} & k_{\perp} \ll k_{c \perp} \\ P_{W} P_{C 0}\left(\frac{k_{n \perp}}{k_{c \perp}}\right)^{q}\left(\frac{k_{c \perp}}{k_{\perp}}\right)^{5 / 3} & k_{\perp} \gg k_{c \perp},\end{cases}$

with

$k_{c \perp}=k_{n \perp} \tau^{-2 /(3-q)}$.

\subsection{Heating rate}

The turbulent wave power is cascaded to smaller scales, until some dissipative process converts the turbulent energy to heat. The heating rate for such process was derived by $\mathrm{Tu}$ (1987) and is given as the sum of the spectral flux at the dissipative scales, $Q_{c}$, and the sweeping effect of radial evolution of the dissipation wave number, $Q_{s}$. The latter effect was found to be small compared to the former in studies of $\mathrm{Hu}$ et al. (1999) and Vainio et al. (2003), when strong cascade is present, and we neglect the sweep effect in this study.

If we assume that the dissipation scales are much smaller than the scale of the spectral break, i.e., $k_{d \perp} \gg k_{c \perp}$, we can write the cascade heating term as

$$
\begin{aligned}
Q_{c \pm} & =\frac{F_{ \pm}\left(k_{d \perp}, r\right)}{4 \pi} \\
& =\frac{1}{4 \pi} \frac{V_{A}}{B} k_{d \perp}^{5 / 2} \alpha_{ \pm}(r, \omega) P_{ \pm}\left(r, k_{d \perp}(r)\right)^{3 / 2} \\
& \approx \frac{1}{4 \pi} \frac{V_{A}}{B} k_{c \perp \pm}^{5 / 2} \alpha(r, \omega)\left[P_{W_{ \pm}} P_{C 0 \pm}\left(\frac{k_{n \perp}}{k_{c \perp \pm}}\right)^{q}\right]^{\frac{3}{2}}
\end{aligned}
$$

where the latter form is derived by using the asymptotic form of Eq. 10, and essentially states that the spectral flux is constant along the inertial range, and can be calculated at the low-wave number limit of the inertial range.. For the heating rate in this paper, we use the sum of heating due to dissipation of both inward and outward waves, $Q_{c}=Q_{c+}+Q_{c-}$.

In order to assert the viability of the model, we must compare the result with observations. To that end, we use the empirical models for the heating rate by Lionello et al. (2001) and Lie-Svendsen et al. (2003) (the first model in their Table 2), who both employ power-law decay, modified by exponential decay at larger heights, of the form

$Q=\frac{F_{m}}{A} \frac{1}{H_{m}} \exp \left\{-\frac{r-r_{1}}{H_{m}}\right\}$,

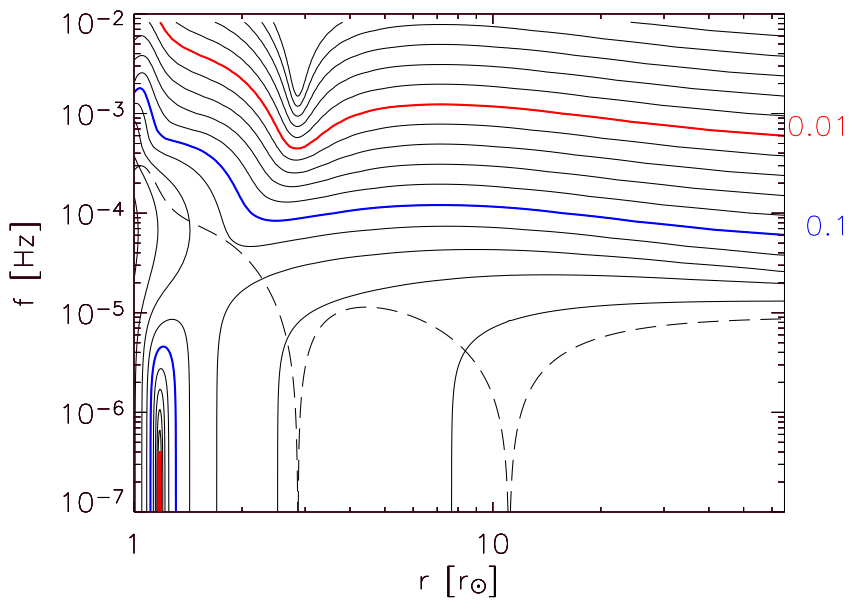

Fig. 2. Contour plot of the parameter $\alpha_{-}$, obtained from the solution of the Elsasser equations, Eq. (2). The plot shows 5 contour lines for each order of magnitude, with values 0.1 and 0.01 shown by the thick blue and red curves, respectively. The values decrease from bottom to top. The dashed line represents the reflection break point frequency, $f_{c}=\omega_{c} / 2 \pi$, given by Eq. (4).

where $F_{m}, H_{m}$ and $r_{1}$ are model parameters. In addition, we use for comparison the heating rates obtained by $\mathrm{Hu}$ et al. (1999), using an 1-dimensional two-fluid code to produce fast solar winds with an ad-hoc cascade rate, parametrized by

$\alpha_{-}=\left\{\begin{array}{lll}0.0625 & \frac{r-r_{\odot}}{9 r_{\odot}} & r_{\odot}<r<10 r_{\odot} \\ 0.0625 & & r \geq 10 r_{\odot} .\end{array}\right.$

All of these models are capable of producing fast solar winds.

\section{Results and Discussion}

In this work, we consider the wave spectrum to composed so that the frequency is considered a parameter for the cascading and heating calculations. The perpendicular wave number spectrum is normalized so that the velocity amplitude is $\left\langle\delta v^{2}\right\rangle^{1 / 2}=30 \mathrm{~km} / \mathrm{s}$ at the coronal base, consistent to line broadening observations in the corona (Hassler et al., 1990; Chae et al., 1998; Doyle et al., 1998). We consider the spectral scales to range from solar radius, $k_{0 \perp}^{-1}=r_{\odot}$ to the thermal proton Larmor radius,

$k_{1 \perp}^{-1}=\sqrt{2 k_{b} T_{p}\left(r_{\odot}\right) m_{p}^{-1}} \frac{m_{p} c}{e B\left(r_{\odot}\right)}$,

for proton temperature $T_{p}=10^{6} \mathrm{~K}$. For the spectral slope, we choose $q=1$ and for the normalization wave numbers arbitrarily $k_{0}=k_{n}=1 \mathrm{~cm}^{-1}$. The frequency normalization, $\omega_{0}$, is canceled out in the $\alpha_{ \pm}$-terms, and is is set to the parametric frequency for each curve in the spectral breakpoint and heating rate figures, so that for a monochromatic frequency spectrum, the integral over the whole spectral range results in the desired $\left\langle\delta b^{2}\right\rangle=B^{2} / V_{A}^{2}\left\langle\delta v^{2}\right\rangle$. 


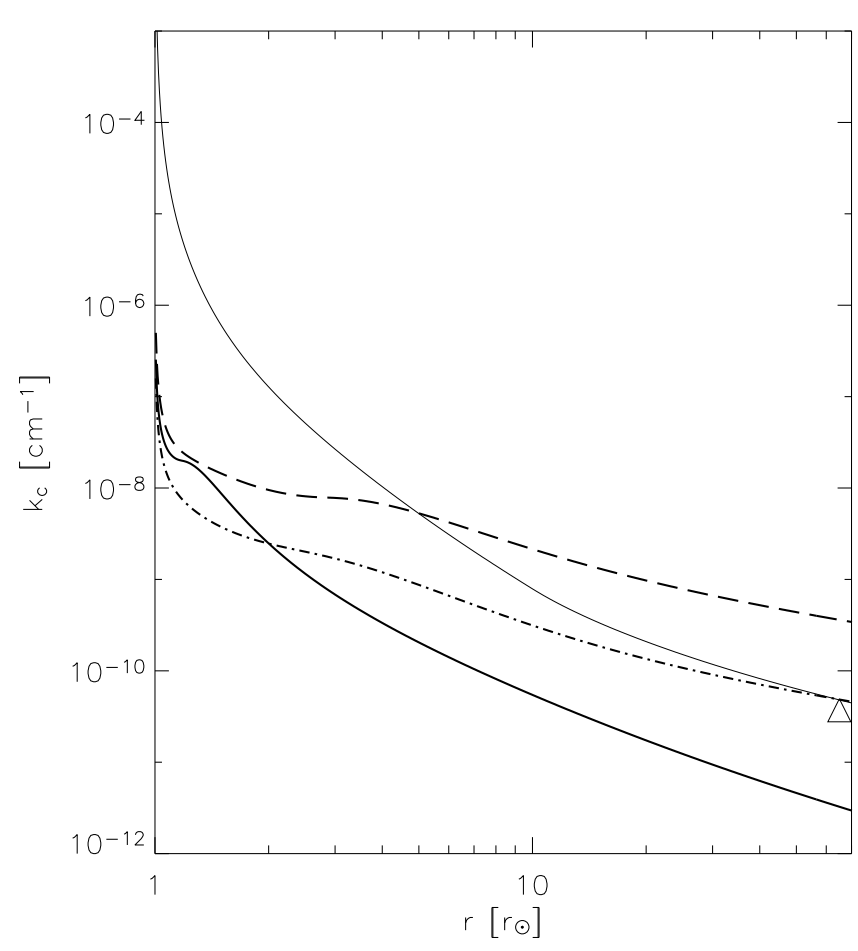

Fig. 3. The thick curves represent the spectral break point wave number of the outward wave spectrum, as a function of distance, for frequencies $10^{-6} \mathrm{~Hz}$ (solid curve), $10^{-4} \mathrm{~Hz}$ (dash-dotted curve) and $10^{-3} \mathrm{~Hz}$ (dashed curve). The solid thin curve shows the spectral break point resulting from the ad-hoc reflection rate, given by Eq. (14). The triangle at the right edge of the figure depicts the spectral breakpoint observed at Helios spacecraft (Tu et al., 1989)

In order to obtain the heating rate, given by Eq. (12), we must first estimate the parameters $\alpha_{ \pm}$. This is done by solving the Elsasser equations, Eqs. (2), and obtaining the power spectral values from the Fourier representation. The resulting ratio $\alpha_{-}=1 / \alpha_{+}$is shown in Fig. 2, as a contour plot. As expected, the ratio is large at low frequencies, whereas at higher frequencies the reflection efficiency decreases and the waves become more predominately outward. The characteristic reflection frequency, $\omega_{c}$, given by Eq. (4) has two local minima, which are located at the Alfvénic point, near $10 r_{\odot}$, and at $2.9 r_{\odot}$, where the Alfvén velocity reaches its maximum and the parameter $R_{A}$ vanishes. The effect of the former minimum is stronger in the high frequencies, where the amount of convected inward waves is smaller and do not shadow the locally decreased reflection. At the Alfvénic point, however, the decrease of reflection is countered by the increase of the WKB factor, $P_{W+}$, of the inward waves. It should be noted that the $\omega_{c}$ does not follow the contour lines well below the Alfvénic point, thus forming an approximative form for $R_{\omega}$ using $\omega$ as a parameter may prove impossible.

After the parameters $\alpha_{ \pm}$are obtained, the spectral breakpoint wave number, $k_{c \perp \pm}$, can be calculated from Eq. (11). The resulting breakpoint wave number for outward propagating waves is presented in Fig. 3 for three $\alpha_{-}$profiles taken from fixed frequency levels. The dependence on the

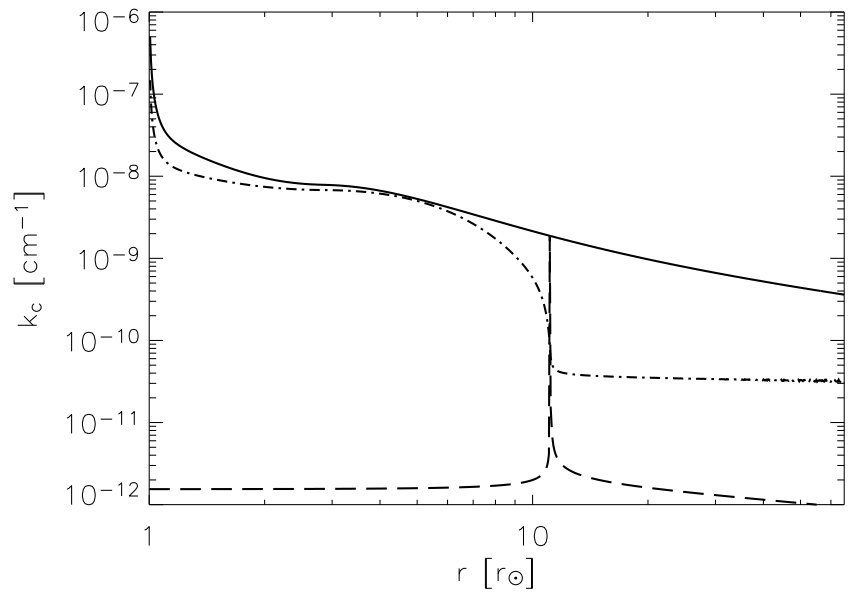

Fig. 4. The spectral breakpoint wave number for frequency $10^{-3} \mathrm{~Hz}$ for outward (solid curve) and inward (dashed curve) waves. The effect of the frequency shift for the outward breakpoint, under the assumption of $P \propto f^{-1}$ spectrum throughout the frequency space, is shown by the dash-dotted curve.

wave frequency is clearly seen, particularly at larger heights, where the difference at these frequencies exceeds an order of magnitude. For comparison, we show the evolution of the spectral breakpoint when using the ad-hoc form of parameter $\alpha$ used in studies of Hu et al. (1999) and Vainio et al. (2003), given by Eq. (14). It is clearly shown that their model produces considerably slower decrease of the spectral break point in the low corona, suggestive of slower heating there. The breakpoint wave number at $10^{-4} \mathrm{~Hz}$ is in relatively good agreement with the breakpoint observed by Helios spacecraft in fast solar wind stream (Tu et al., 1989).

We also show the effect of the frequency shift, discussed above, due to the reflection conserving the fixed frame frequency, whereas the wave-wave interactions work between waves of the same scale. The dot-dashed curve in Fig. 4 shows the effect of this shift, under the assumption that the frequency spectrum of the waves extends to very large scales with a $P \propto f^{-1}$ spectrum. As can be seen, near the Alfvénic point the breakpoint wave number decreases considerably, suggesting very strong spectral flux, and thus also strong heating. However, as the nature of the spectrum at very large scales, needed for the generation of the interacting reflected waves, is not known well, and not likely to continue the $f^{-1}$ trend to very low frequencies, we find it not very reliable to estimate the heating in this manner at this point.

The heating rate of the solar wind plasma by dissipation of the turbulence at small scales, $k_{d \perp} \gg k_{c \perp}$, is shown in Fig. 5, for three frequencies. For comparison, we also present the Lionello et al. (2001) and Lie-Svendsen et al. (2003) empirical heating rates, and the heating rate from the solar wind model by $\mathrm{Hu}$ et al. (1999). The heating rate of $\mathrm{Hu}$ et al. (1999) follows the modeled heating rates quite nicely, except for the above discussed heating reduction at higher frequencies, and a narrow shell of about $0.1 r_{\odot}$, where the heating in our model is considerably strong. However, interestingly, 


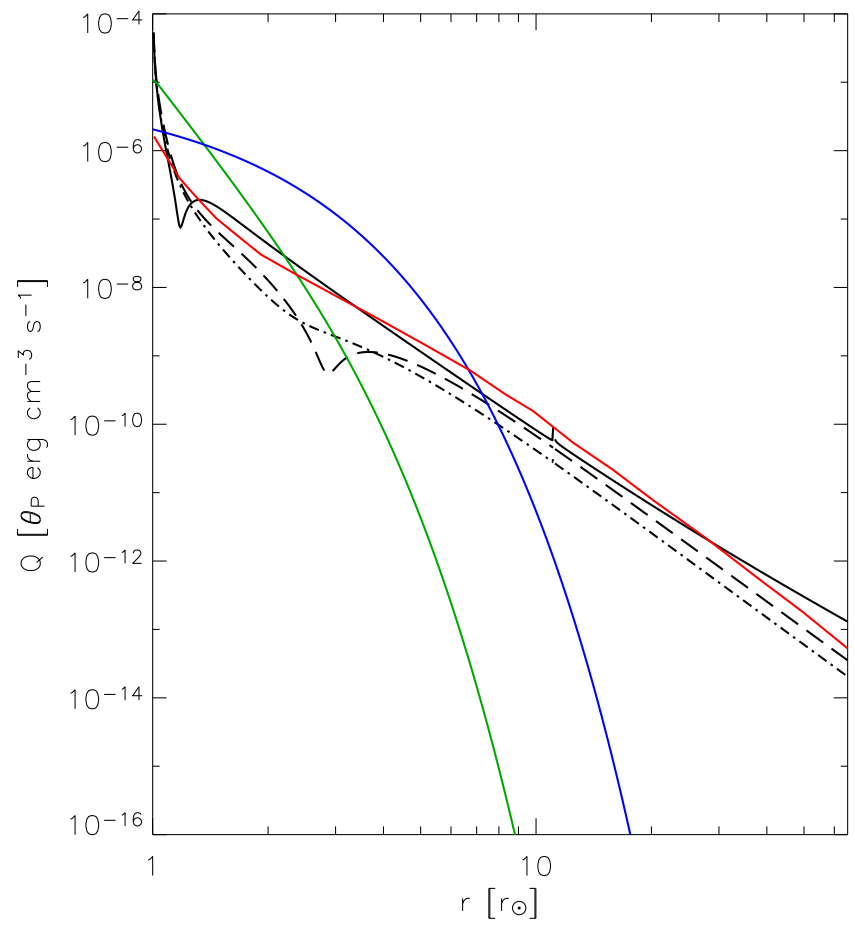

Fig. 5. Heating rates for a cascade due to consistent cascading at frequencies $10^{-6} \mathrm{~Hz}$ (solid curve), $10^{-4} \mathrm{~Hz}$ (dash-dotted curve) and $10^{-3} \mathrm{~Hz}$ (dashed curve). The Lionello et al. (2001) and LieSvendsen et al. (2003) empirical heating rates are shown by the blue and green curves, respectively. The heating rate of $\mathrm{Hu}$ et al. (1999) model, obtained by the ad-hoc cascade rate of Eq. (14), is shown by the red curve.

the heating rate models by Lionello et al. (2001) and LieSvendsen et al. (2003) seem quite different, as they heat the corona at considerably higher rate at low altitudes, but then decrease exponentially quite fast above $\sim 10 r_{\odot}$. It should be noted, though, that all of the four cited studies produce fast wind streams, but hold different levels of physics in their solar wind models, and concentrate on different issues.

The effect of the frequency in the heating rate ranges from 0.5 to 1 order of magnitude, depending on the radial distance from the coronal base. The effect is at its strongest at around $3 r_{\odot}$, which is the region where the reflection is reduced due to the local maximum of the Alfvén velocity. This region is particularly interesting in terms of solar wind heating and acceleration, as it is close to the usual region of the sonic point. As discussed by Leer et al. (1982), heating the subsonic wind increases the plasma temperature and mass flux, whereas in the supersonic wind the energy increase accelerates the solar wind to faster velocities. Therefore, the influence of the frequency spectrum of the turbulence may prove important in modeling the solar wind.

It should be noted, that the requirement for the independence of the parameters $\alpha_{ \pm}$of the perpendicular wave number, $k_{\perp}$, is only an approximation, and this should be remembered when estimating the accuracy of this simple heating rate model. Observations in the interplanetary space suggest that the spectral breakpoints for the inward and outward spectra may differ (e.g. Tu et al., 1989). In Fig. 4, we show the evolution of the inward spectral breakpoint (dashed curve), assuming that the it coincides with the outward spectral break at Alfvénic point. While the inward and outward breakpoints differ in this presentation considerably, it is expected that the reflected outward spectrum, characterised locally by the outward breakpoint (solid curve), modifies the inward spectrum, bringing the breakpoint to higher wave numbers. The estimation of the balance between reflection and cascading on the inward spectrum requires further numerical work.

The study presented in this paper only considers excitation of the waves on single frequencies, whereas a more consistent approach would consider a finite bandwidth of turbulence injection. Scintillation observations suggest that close to the Sun, below $10 r_{\odot}$, the turbulence spectrum follows a power-law of $f^{-1}$, over a at least the frequency range of $10^{-3}-10^{1} \mathrm{~Hz}$, but becomes more Kolmogorov-like at larger heliocentric distances (Woo and Armstrong, 1979). These observations, however, depict density fluctuations, and the connection between such observations and the different wave modes, particularly with the non-compressive Alfvén waves studied here, is not well known. In the interplanetary space, the observed magnetic fluctuations also show similar $f^{-1}$ structure at low frequencies, breaking into Kolmogorov-like spectrum at higher wave numbers (e.g. Tu et al., 1989; Klein et al., 1992; Horbury et al., 1996). In the corona, the higher frequencies, required for ion cyclotron heating, which is supported by temperature anisotropy observations (Kohl et al., 1998), are beyond current observational capabilities.

The effect of a wide frequency spectrum to the solar wind heating is difficult to estimate, as it depends strongly on the efficiency of dissipation by different mechanisms for different frequencies and wave numbers. If we assume that the dissipation occurs only at small perpendicular scales, with no frequency dependence, we can consider the lowest frequency available to dominate the heating, as, particularly for a decreasing frequency spectrum, the heating rate decreases rapidly as a function of frequency above $10^{-5} \mathrm{~Hz}$ (cf. Figs. 2 and 5). Assuming that the maximum of the excitation takes place isotropically at the turbulence outer scale of roughly $0.2 r_{\odot}$ (e.g. Efimov et al., 2004), i.e. $k=7 \cdot 10^{-11} \mathrm{~cm}^{-1}$, this dominating frequency would be $3 \cdot 10^{-3} \mathrm{~Hz}$, consistent also with Faraday rotation fluctuation observations (Efimov et al., 1993). One should, however, bear in mind that the observed spectral evolution acts as a constraint with respect to the solar wind heating modeling, as the observations suggest that the turbulence is still developing higher in the corona (Woo and Armstrong, 1979), and also in the interplanetary space, at distances $>0.3 \mathrm{AU}$ (Bavassano et al., 1982). In addition, as the turbulence affects the motion of energetic particles, the turbulence modeling, and subsequently the solar wind modeling, is also constrained by observations of acceleration and interplanetary transport of solar energetic particles (e.g. Vainio and Laitinen, 2001).

The study presented in this paper considers the spectral evolution to take place solely on the direction perpendicular 
to the magnetic field. As shown by Goldreich and Sridhar (1995), albeit the perpendicular spectral flux is dominant in the presence of background magnetic field, there is also a finite flux to the parallel direction. In addition, as recent modeling results suggest, different wave modes evolve differently (Cho and Lazarian, 2003), and the isotropic cascade of fast mode waves may come into question when interpreting the observations. Modeling of the cascade in parallel direction consistently with the presented non-WKB reflections, however, requires more rigorous treatment.

\section{Conclusions}

In this study, we have reviewed the phenomenological turbulence cascading model and the resulting heat deposition into the corona, by developing a simple, yet more consistent description for the non-WKB reflection of the waves from the solar wind background gradients. The study was limited to perpendicular cascading, and no specific dissipation mechanism was presented.

We found that the cascade advances fast in the low corona, and has a strong frequency dependence. Also the heating rate was found to have a strong frequency dependence, particularly in the low corona. We find that these result suggest the shape and range of the frequency spectrum of the turbulence has important implications on the solar wind modeling. Further work, in both more consistent work on the spectral evolution, and incorporation of solar wind and proper dissipation models are needed to better quantify these effects.

\section{Appendix A Semi-Analytic solution method for the Elsasser equations}

In this Appendix we present a semi-analytical solution for the Elsasser equation, Eq. (2). The method follows closely the one presented by Heinemann and Olbert (1980), but is applied to the Elsasser variables used in this study.

It is often convenient to use WKB units with $z_{\phi}^{ \pm}=z_{C}^{ \pm} z_{W}^{ \pm}$ to simplify the Eq. (2) to

$\frac{\partial z_{C}^{ \pm}}{\partial t}+V_{\mp} \frac{\partial z_{C}^{ \pm}}{\partial r}+V_{\mp} R_{A} z_{C}^{\mp}=0$.

The equation can be solved semi-analytically for toroidal waves of form $z_{C}^{ \pm}(r, t)=z_{0}^{ \pm}(r, \omega) \exp \{-i \omega t\}$. For such fields we can write

$-i \omega z_{C}^{ \pm}+V_{\mp} \frac{\partial z_{C}^{ \pm}}{\partial r}+V_{\mp} R_{A} z_{C}^{\mp}=0$.

The first term on the left hand side can be eliminated using $z_{C}^{ \pm}=z_{0}^{ \pm} \exp \left\{i \omega \tau_{\phi \pm}\right\}$, where $d \tau_{\phi_{ \pm}}=d r / V_{\mp}$, and $d \tau_{\phi}=d \tau_{\phi-}-d \tau_{\phi_{+}}$, with $\tau_{\phi}$ the transit time difference between the $z_{0}^{ \pm}$variables, to yield

$\frac{\partial z_{0}^{ \pm}}{\partial r}+z_{0}^{\mp} R_{A} e^{ \pm i \omega \tau_{\phi}}=0$ or

$\frac{\partial z_{0}^{ \pm}}{\partial \tau_{\phi}}=\frac{U^{2}-V_{A}^{2}}{2 V_{A}} z_{0}^{\mp} W e^{ \pm i \omega \tau_{\phi}}$

This can then be solved formally to give

$z_{0}^{ \pm}=z_{00}^{ \pm}-\int z_{0}^{\mp} \frac{V_{A}^{2}-U^{2}}{2 V_{A}} \frac{\partial}{\partial r} \ln \left\{\sqrt{\frac{A}{V_{A}}}\right\} e^{ \pm i \omega \tau_{\phi}} d \tau_{\phi}$,

which can be solved iteratively using numerical methods designed for Fourier integrals and appropriate boundary conditions.

The equation has a critical point at the Alfvénic point, which in $\tau_{\phi}$-variable, and the counterstreaming wave WKB solution, $z_{W}^{+}$, approaches infinity. As the Alfvénic point is a divergent point for the inward waves, the power of those waves in that point must be zero, since no waves are generated there. Thus, the boundary can be set at the Alfvénic point, with $z_{0}^{+}\left(r_{A}\right)=0$ and $z_{0}^{-}\left(r_{A}\right)=z_{00}^{-}$.

It should be noted that as the +-component diverges at the Alfvénic point, it is convenient to perform the integrations starting from the Alfvénic point.

Acknowledgements. The author acknowledges the financial support of the Academy of Finland, projects 204026 and 207649, and the EU training network PLATON (EC contract HPNR-CT-200000153). The author also thanks H. Fichtner, R. Kissmann and R. Vainio for valuable discussions. The referees are acknowledged for their input, which improved the paper considerably.

Edited by: S. E. Ferreira

Reviewed by: two referees

\section{References}

Axford, W. I. and McKenzie, J. F.: The origin of high speed solar wind streams, in Solar Wind Seven; Proceedings of the 3rd COSPAR Colloquium, 1-5, 1992.

Barkhudarov, M. R.: Alfvén waves in stellar winds, Sol. Phys., 135, 131-161, 1991.

Bavassano, B., Dobrowolny, M., Mariani, F., and Ness, N. F.: Radial evolution of power spectra of interplanetary Alvénic turbulence, J. of Geophys. Res., 87, 3617-3622, 1982.

Chae, J., Schühle, U., and Lemaire, P.: SUMER Measurements of Nonthermal Motions: Constraints on Coronal Heating Mechanisms, Astrophys. J., 505, 957-973, 1998.

Cho, J. and Lazarian, A.: Compressible magnetohydrodynamic turbulence: mode coupling, scaling relations, anisotropy, viscositydamped regime and astrophysical implications, Mon. Not. of the Roy. Astron. Soc., 345, 325-339, 2003.

Cho, J. and Vishniac, E. T.: The Anisotropy of Magnetohydrodynamic Alfvnic Turbulence, Astrophys. J., 539, 273-282, 2000.

Cranmer, S. R.: Ion Cyclotron Wave Dissipation in the Solar Corona: The Summed Effect of More than 2000 Ion Species, Astrophys. J., 532, 1197-1208, 2000.

Cranmer, S. R.: Coronal Holes and the High-Speed Solar Wind, Space Science Rev., 101, 229-294, 2002.

Cranmer, S. R. and van Ballegooijen, A. A.: Alfvnic Turbulence in the Extended Solar Corona: Kinetic Effects and Proton Heating, Astrophys. J., 594, 573-591, 2003. 
Cranmer, S. R. and van Ballegooijen, A. A.: On the Generation, Propagation, and Reflection of Alfvén Waves from the Solar Photosphere to the Distant Heliosphere, Astrophys. J. Suppl. Ser., 156, 265-293, 2005.

Doyle, J. G., Banerjee, D., and Perez, M. E.: Coronal line-width variations, Sol. Phys., 181, 91-101, 1998.

Efimov, A. I., Chashei, I. V., Shishov, V. I., and Bird, M. K.: Faraday rotation fluctuations during radio transillumination of circumsolar plasma, Astron. Let., 19, 57-61, 1993.

Efimov, A. I., Bird, M. K., Chashei, I. V., and Samoznaev, L. N.: Outer scale of solar wind turbulence deduced from two-way coronal radio sounding experiments, Adv. in Space Res., 33, 701-706, 2004.

Elsasser, W. M.: The Hydromagnetic Equations, Phys. Rev., 79, $183,1950$.

Goldreich, P. and Sridhar, S.: Toward a theory of interstellar turbulence. 2: Strong alfvenic turbulence, Astrophys. J., 438, 763-775, 1995.

Hassler, D. M., Rottman, G. J., Shoub, E. C., and Holzer, T. E.: Line broadening of MG X 609 and 625 A coronal emission lines observed above the solar limb, Astrophys. J., 348, 77-80, 1990.

Heinemann, M. and Olbert, S.: Non-WKB Alfvén waves in the solar wind, J. of Geophys. Res., 85, 1311-1327, 1980.

Hollweg, J. V.: Transition region, corona, and solar wind in coronal holes, J. of Geophys. Res., 91, 4111-4125, 1986.

Horbury, T. S., Balogh, A., Forsyth, R. J., and Smith, E. J.: The rate of turbulent evolution over the Sun's poles., Astron. and Astrophys., 316, 333-341, 1996.

$\mathrm{Hu}$, Y. Q., Habbal, S. R., and Li, X.: On the cascade processes of Alfvén waves in the fast solar wind, J. of Geophys. Res., 104, 24 819-24 834, 1999.

Klein, L. W., Matthaeus, W. H., Roberts, D. A., and Goldstein, M. L.: Evolution of spatial and temporal correlations in the solar wind - Observations and interpretation, in Solar Wind Seven; Proc. of the 3rd COSPAR Coll., 197-200, 1992.

Kohl, J. L., Noci, G., Antonucci, E., Tondello, G., Huber, M. C. E., Cranmer, S. R., Strachan, L., Panasyuk, A. V., Gardner, L. D., Romoli, M., Fineschi, S., Dobrzycka, D., Raymond, J. C., Nicolosi, P., Siegmund, O. H. W., Spadaro, D., Benna, C., Ciaravella, A., Giordano, S., Habbal, S. R., Karovska, M., Li, X., Martin, R., Michels, J. G., Modigliani, A., Naletto, G., O’Neal, R. H., Pernechele, C., Poletto, G., Smith, P. L., and Suleiman, R. M.: UVCS/SOHO Empirical Determinations of Anisotropic Velocity Distributions in the Solar Corona, Astrophys. J., 501, 127-131, 1998.

Leer E., Holzer, T. E., and Flå, T.: Acceleration of the solar wind, Space Science Rev., 33, 161-200, 1982.

Lie-Svendsen, O., Hansteen, V. H., and Leer, E.: Helium Abundance in the Corona and Solar Wind: Gyrotropic Modeling from the Chromosphere to 1 AU, Astrophys. J., 596, 621-645, 2003.

Lionello, R., Linker, J. A., and Mikić, Z.: Including the Transition Region in Models of the Large-Scale Solar Corona, Astrophys. J., 546, 542-551, 2001.
Marsch, E. and Mangeney, A.: Ideal MHD equations in terms of compressive Elsaesser variables, J. of Geophys. Res., 92, 7363-7367, 1987.

Marsch, E. and Tu, C.-Y.: Dynamics of correlation functions with Elsasser variables for inhomogeneous MHD turbulence, J. of Plasma Phys., 41, 479-491, 1989.

Marsch, E. and Tu, C.-Y.: Evidence for pitch angle diffusion of solar wind protons in resonance with cyclotron waves, J. of Geophys. Res., 106, 8357-8362, 2001.

Matthaeus, W. H., Zank, G. P., Oughton, S., Mullan, D. J., and Dmitruk, P.: Coronal Heating by Magnetohydrodynamic Turbulence Driven by Reflected Low-Frequency Waves, Astrophys. J., 523, 93-96, 1999.

Parker, E. N.: Dynamics of the Interplanetary Gas and Magnetic Fields., Astrophys. J., 128, 664-676, 1958.

Shebalin, J. V., Matthaeus, W. H., and Montgomery, D.: Anisotropy in MHD turbulence due to a mean magnetic field, J. of Plasma Phys., 29, 525-547, 1983.

Tu, C.-Y.: A solar wind model with the power spectrum of Alvénic fluctuations, Sol. Phys., 109, 149-186, 1987.

Tu, C.-Y.: The damping of interplanetary Alvénic fluctuations and the heating of the solar wind, J. of Geophys. Res., 93, 7-20, 1988.

Tu, C.-Y. and Marsch, E.: MHD structures, waves and turbulence in the solar wind: Observations and theories, Space Science Rev., 73, 1-210, 1995.

Tu, C.-Y. and Marsch, E.: Two-Fluid Model for Heating of the Solar Corona and Acceleration of the Solar Wind by High-Frequency Alfvén Waves, Sol. Phys., 171, 363-391, 1997.

Tu, C.-Y., Pu, Z.-Y., and Wei, F.-S.: The power spectrum of interplanetary Alvénic fluctuations Derivation of the governing equation and its solution, J. of Geophys. Res., 89, 9695-9702, 1984.

Tu, C.-Y., Marsch, E., and Thieme, K. M.: Basic properties of solar wind MHD turbulence near 0.3 AU analyzed by means of Elsaesser variables, J. of Geophys. Res., 94, 11 739-11 759, 1989.

Vainio, R. and Laitinen, T.: The relation between cyclotron heating and energetic particles on open coronal field lines, Astron. Astrophys., 371, 738-747, 2001.

Vainio, R., Laitinen, T., and Fichtner, H.: A simple analytical expression for the power spectrum of cascading Alfvén waves in the solar wind, Astron. Astrophys., 407, 713-723, 2003.

Verdini, A., Velli, M., and Oughton, S.: Nonlinear Evolution of a Turbulent Spectrum of Outwardly Propagating Alfvén Waves in Solar and Stellar Coronae, in ESA SP-575: SOHO 15 Coronal Heating, 15, 454-459, 2004.

Woo, R. and Armstrong, J. W.: Spacecraft radio scattering observations of the power spectrum of electron density fluctuations in the solar wind, J. of Geophys. Res., 84, 7288-7296, 1979.

Zhou, Y. and Matthaeus, W. H.: Models of inertial range spectra of interplanetary magnetohydrodynamic turbulence, J. of Geophys. Res., 95, 14 881-14 892, 1990a.

Zhou, Y. and Matthaeus, W. H.: Transport and turbulence modeling of solar wind fluctuations, J. of Geophys. Res., 95, 10291-10311, 1990b. 